\title{
reprise des fondations sur zone de décharge
}

\author{
par \\ D. Gouvenot \\ SOLETANCHE-Entreprise
}

RESUME - La construction de bâtiments sur zone de décharge pose des problèmes de fondations très particuliers. Les plus marquants sont la compacité très faible des formations rencontrées, et les processus chimiques de décomposition des matières qui provoquent des tassements incontrôlables. De nombreux sinistres ont résulté de ces difficultés. Il a fallu reprendre en sous oeuvre les bâtiments endommagés, par une reprise de fondations à base de pieux injectés métalliques (pieux IM). Des protections contre la corrosion, très soignés, sont prévues car on se trouve souvent en milieu agressif. Les justifications géotechniques de ces fondations sont particulières et nécessitent des essais de contrôle.

\section{INTRODUCTION}

Il n'est pas rare de voir construire, sur des zones de décharge, de grands ensembles industriels ou commerciaux. Il s'agit souvent de terrains situés au voisinage des villes, en général bon marché, ce qui provoque un attrait certain pour le constructeur et explique, sans doute, la volonté de ce dernier de vouloir construire sur des sites qui, sur le plan de la technique, présentent un très grand nombre de difficultés. Il s'agit en effet, pour le géotechnicien, de définir une fondation dans des formations qui ont des propriétés extrêmement particulières. Au niveau de la compacité, les hétérogénéités sont très grandes puisque $1^{\prime}$ on peut aussi bien rencontrer des déblais de démolition que des chiffons, des pièces de voitures, etc... La compacité est donc très variable dans l'espace mais aussi dans le temps, puisque nombre de matériaux, d'origine organique, peuvent subir des processus de décomposition chimique. Ces considérations simples expliquent déjà les nombreuses difficultés rencontrées sur les ouvrages fondés superficiellement. Ces désordres, qui ne sont même pas liés au taux de travail des semelles par exemple, sont inhérents à la nature même du sol de fondation, si on peut 1 'appeler ainsi. Les techniques de fondations profondes qui, sur le plan de la géotechnique, répondent mieux à la question, puisqu'il est alors possible de reporter les charges de la structure au-delà de la hauteur du remblai, dans des couches de sol saines, posent par contre des problèmes d'exécution très sérieux. En effet, il n'est pas rare de rencontrer aléctoire- ment des obstacles infranchissables, même au trépan, tels que carrosseries de voitures ou pièces de bois. Ces considérations expliquent que nous ayons été amenés à intervenir sur plusieurs types de sinistres dont nous ne citerons que deux exemples parmi les plus représentatifs:

$1^{\circ}$ - Dans le premier cas, il s'agit d'un bâtiment industriel fondé superficiellement.

$2^{\circ}$ - Dans le second cas, il s'agit d'un bâtiment à usage d'habitation fondé sur pieux forés, dont la base, par suite d'obstacles infranchissables, se trouve encore dans les remblais de décharge au lieu de rejoindre les couches de sol sain sous-jacent.

Dans les deux cas, les reprises de fondations ont été effectuées à 1 'aide du pieu injecté métallique IM, et ont permis de sauver les bâtiments.

\section{Définition du pieu IM}

Ce pieu a été déjà décrit par Messieurs FENOUX (1976) et GOUVENOT (1975). Il consiste en une armature métallique (en général un tube dont le diamètre varie entre 100 et 200 $\mathrm{mm})$ mise en place dans un forage, puis scellée au sol par injection de ciment sous pression. Ceci lui confère des capacités portantes très élevées (jusqu'à 200 tonnes) qui ont été vérifiées par de nombreux essais déjà publiés (GOUVENOT, 1973 et GOUVENOT, 1974). L'avantage du procédé, dans le cas particulier qui nous préoccupe, est qu'il s'agit 
d'abord d'une technique de reprise en sous oeuvre qui nécessite des forages de petit diamètre, et qui se joue donc plus facilement des obstacles que $1^{\prime}$ on peut rencontrer dans un remblai de décharge. De plus, sur ce petit diamètre, il est possible de faire passer des efforts axiaux importants par $1^{\prime} u-$ tilisation de tubes métalliques aux nuances élevées. Un autre avantage est offert par l'injection de ciment. Le coulis peut assurer un remplissage des vides. On a même utilisé des coulis de ciment comme fluide de perforation pour améliorer le remblai. En cas d'obstacles infranchissables, il est plus facile de modifier l'implantation du pieu IM. Les adaptations de longrines qui en résultent sont moins gênantes que pour un pieu de gros diamètre.

\section{ler cas de réalisation}

Il s'agit de la reprise en sous oeuvre des fondations d'un immeuble de 24 niveaux fondé sur un remblai de décharge, à l'aide de pieux forés dont la base n'avait pas pu atteindre les couches de calcaire situées à la base du remblai. Lors de la construction, des tassements différentiels de 1 'ordre de $15 \mathrm{~cm}$ ont conduit à des désordres très graves. Il a fallu exécuter, à partir du dernier sous-sol, environ 780 pieux nécessaires pour reprendre le poids total de l'immeuble estimé à 50000 tonnes environ. La charge nominale des pieux IM, comprise entre 50 et 70 tonnes, était assurée par des armatures à base d'acier TOR scellées dans les couches de sol sous-jacentes au remblai.

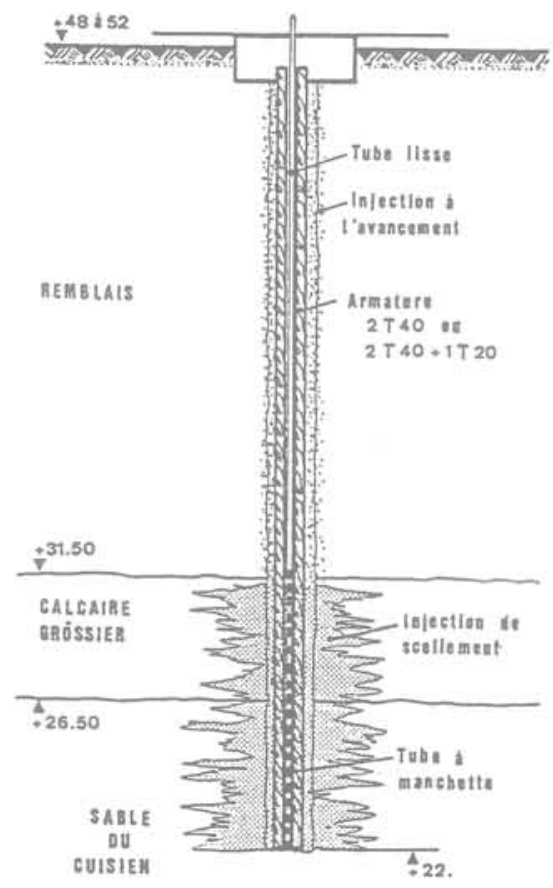

Figure 1 : Pieu IM de 50 à 70 tonnes de capacité à travers un remblai de décharge
Le diamètre des forages $(15 \mathrm{~cm}$ ) a permis de s'affranchir complètement des obstacles et 1 'effet de 1 'injection sous pression sur la qualité du scellement a été vérifié par des essais de traction. La rapidité d'exécution a permis de sauver le bâtiment. La protection contre la corrosion a été assurée par enduction de brai sur l'armature métallique, de plus, une gaine de coulis de ciment protège l'ensemble du pieu IM sur toute sa hauteur.

\section{2 e cas de réalisation}

Un bâtiment industriel avait été construit sur semelles superficielles. Comme ces semelles surmontaient environ $6 \mathrm{~m}$ de déchets ménagers, le projeteur avait prévu de faire reposer ces semelles sur des colonnes balastées qui traversaient le remblai, pour se poser sur les alluvions du Rhône sous-jacentes. On ne discutera pas ici de la qualité de ces colonnes, toujours est-il qu'après deux ans d'exploitation, le bâtiment a vu ses semelles tasser à un point tel, que la charpente métallique fut soumise à des sollicitations inacceptables. Il fut décidé de reprendre ce bâtiment, en sous oeuvre, par des injections, mais les vides étaient trop importants et, d'autre part, lors de l'exécution des forages nécessaires pour réaliser les travaux d'injection, des dégagements gazeux nauséabonds furent décelés, signe évident de la décomposition des déchets ménagers. Dans ces conditions, toute solution, qui cherchait à améliorer la compacité du remblai, ne pouvait avoir qu'un effet provisoire. Il fallait donc reporter les charges dans les alluvions du Rhône à l'aide de pieux. La technique du pieu IM fut retenue car, outre les avantages déjà cités précédemment, elle permettait de travailler dans des ateliers en n'occupant que des zones de travail limitées, en raison des dimensions réduites de la machine de perforation utilisée.

La reprise en sous oeuvre a été réalisée de la manière suivante (figure 2):

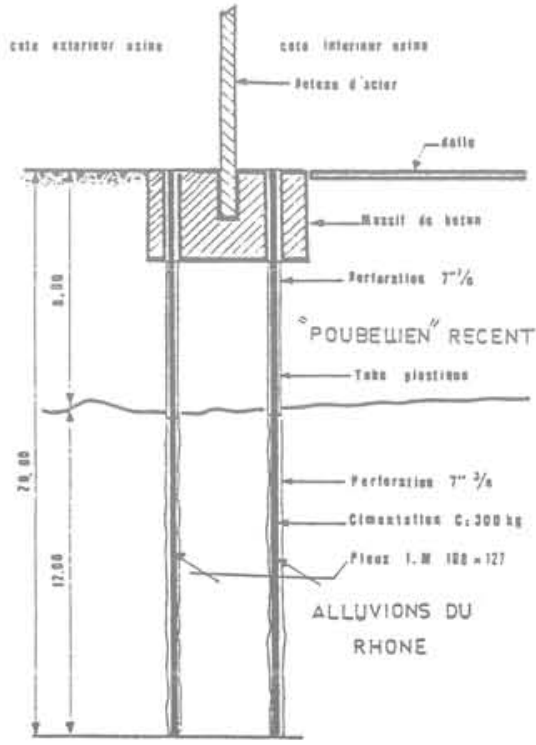

Figure 2 :

Pieux IM tubulaires de 100 tonnes d̀ travers une zone de décharge ménagère 
$1^{\circ}$ - Exécution d'un pré-forage à travers le béton des semelles.

$2^{\circ}$ - Forage dans le remblai de déchets ménagers et mise en place d'un tube destiné à soutenir le forage et à protéger le pieu à travers le remblai.

$3^{\circ}$ - Forage sur 12 mètres de hauteur dans les alluvions du Rhône.

$4^{\circ}$ - Mise en place du tube métallique de $13 \mathrm{~cm}$ de diamètre constituant 1 'armature du pieu IM, de capacité unitaire 100 tonnes. Le pieu est protégé à travers le remblai par un revêtement brai époxy.

$5^{\circ}$ - Scellement du pieu à la semelle en béton.

$6^{\circ}$ - Injection de coulis de ciment dans les alluvions pour assurer le scellement du pieu au sol.

Comme dans le cas précédent, des essais de chargement ont été réalisés pour vérifier la stabilité élastique du pieu sous la charge de service (problème de flambement et capacité du scellement réalisé dans les alluvions). Notons que le flambement a également été examiné sur le plan théorique en supposant que la décomposition du remblai se poursuivait et que le pieu IM pouvait se trouver totalement dégagé sur plusieurs mètres de hauteur.

La figure 3 montre le dispositif utilisé pour charger simultanément deux pieux d'essai en assurant la reprise des efforts par la structure du bâtiment lui-même.

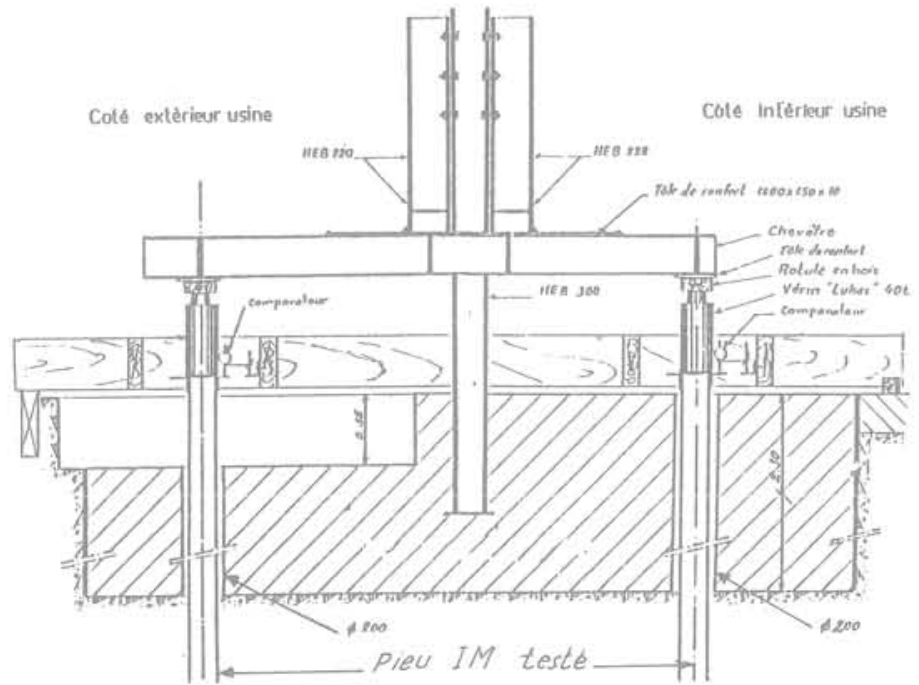

Figure 3 : Essai de chargement simultané de deux pieux IM

La figure 4 montre la courbe de chargement obtenue, la charge axiale ayant été volontairement ramenée à 25 tonnes pour les problèmes de flambement évoqués ci-dessus.
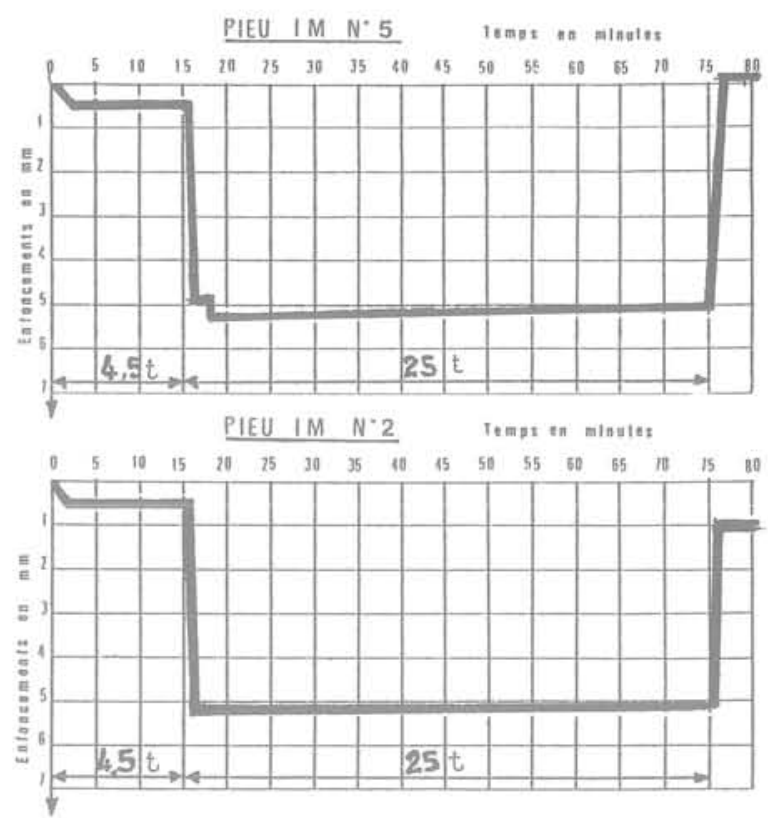

Figure 4: Résultats d'essai de chargement à 25 tonnes (charge de service)

\section{CONCLUSION}

D'une façon générale, la facilité de mise en oeuvre des pieux IM ainsi que leurs propriétés mécaniques en indiquent l'emploi:

- Chaque fois qu'il y a risque de rencontre d'obstacles tels que : anciennes fondations, blocs, couches dures, etc... qu'il serait très onéreux ou impossible de traverser en forage de grande section.

- Toutes les fois que l'on doit, au départ, traverser des massifs de maçonnerie ou de béton sans ébranler les structures qui sont souvent voisines de la ruine.

- Quand I'espace disponible est réduit au point que la manoeuvre du matériel de forage classique est difficile, voire impossible.

Dans tous ces cas que l'on rencontre en matière de reprise en sous oeuvre et pas seulement sur les zones de décharge, la technique du pieu IM a permis de sauver des bâtiments de très grande valeur qui étaient très proches de la ruine. Les protections $v$ is-à-vis de la corrosion et du flambement sont bien au point et les contrôles de capacité sont faciles à réaliser car ils peuvent être conduits aussi bien en compression qu'en traction, donc dans des conditions beaucoup plus aisées que pour les pieux de gros diamètre. 


\section{REFERENCES}

Fenoux GY., (1976). Les pieux aiguilles IM. Construction $n^{\circ} 6$ juin 1976.

Gouvenot D., (1973). Essais en France et à

l'Etranger sur le frottement latéral en

fondation - Amélioration par injections.

Travaux novembre 1973.

Gouvenot D., (1974). Essais de pieux scellés par injection sous pression.

Annales de I'ITBTP novembre 1974.

Gouvenot D., (1975). Essais de chargement

et de flambement de pieux aiguilles.

Annales de l'ITBTP décembre 1975. 